\title{
Astronomy
}

\section{The Geminga enigma unfolds}

from A.W. Strong

THE $\gamma$-ray source CG195 + 04 ('Geminga') discovered by the SAS-2 satellite and observed repeatedly by COS-B is the second brightest source in the COS-B catalogue; unlike the other two bright sources (the Vela and Crab pulsars) it has resisted many attempts to find a convincing counterpart at other wavelengths. At a meeting held by the Royal Astronomical Society in May, G. Bignami (CNR, Milan) reported observations made in collaboration with P. Caraveo (CNR, Milan) and R. Lamb (Iowa State University) that suggest they have been able to identify $X$ rays from the same source, and may at last be on the way to resolving the enigma.

The basic problem has always been the large radius of the $\gamma$-ray error box (radius $0.4^{\circ}$ ) which makes optical searches meaningless, there being no bright or obviously unusual object in the field. Using the imaging proportional counter (IPC) on the Einstein X-ray Observatory the authors found four sources in or near the error circle. Three are compatible with the serendipitous sample expected for the limiting flux; one can be identified with a star of typical X-ray to optical flux ratio, another with a faint (probably extragalactic) radio source, the third is unidentified. The fourth X-ray source, by far the brightest, was subsequently observed with the Einstein high-resolution imager (HRI), allowing a position to be determined to within 3 arc $s$. The corresponding position is blank on the Palomar sky survey; a subsequent CCD image from the European Southern Observatory revealed a blue object of about twenty-third magnitude, presumably the counterpart of the X-ray source. Meanwhile deep pulsar searches at Arecibo gave negative results, and observations with the very large array (VLA) telescope give an upper limit of $1 \mathrm{mJy}$ for any source at $6-\mathrm{cm}$ wavelength.

A critical clue to the physical nature of the X-ray source comes from the X-ray spectrum as determined by the IPC; it is extremely steep betwen 0.3 and $1 \mathrm{keV}$, which implies very little interstellar absorption (assuming a power-law or thermal spectrum at the source), and allows an upper limit on the distance of about $200 \mathrm{pc}$ to be determined. The only alternative would be a line at $260 \mathrm{eV}$ (with arbitrary absorption), which is also consistent with the data. No variation, periodic or otherwise, has been found for the X-ray source.

Various facts suggest the $\mathrm{X}$-ray source is unique: the high X-ray to optical flux ratio $(\sim 1,000)$, the point-like appearance in the $\mathrm{HRI}$ and the absence of a radio source in the VL.A survey are properties not shared by any other Einstein source known. It is this unusual nature which makes the sug- gestion of an association with the $\gamma$-ray source convincing, even though a strict proof is so far impossible.

The observations place severe constraints on the nature of Geminga. The $\mathrm{X}$-ray to optical flux ratio rules out cataclysmic variables, white dwarfs and other hot stars. Only low-mass binary stars and radio pulsars have such high ratios, and the binary stars are ruled out because a distance of $250 \mathrm{kpc}$ would be necessary to account for the faint apparent magnitude, and this is quite inconsistent with the limit from interstellar absorption as well as being far outside the Galaxy.

The remaining possibilities considered by the authors is a pulsar or just an unpulsing neutron star.

The pulsar hypothesis is attractive in view of the general similarity of the source to the Vela pulsar at X-ray and $\gamma$-ray wavelengths, and the lack of a radio signal could in principle be assigned to different beaming geometries in $\gamma$-ray and radio wavelengths. But the lack of an extended $\mathrm{X}$-ray source shows that it is not associated with a 'synchrotron nebula' of the type seen around other X-ray pulsars. The lack of a supernova remnant characteristic of fast X-ray-emitting pulsars also argues against the pulsar hypothesis.

Thus by a process of elimination they arrive at the neutron star. At a distance of 100 pc the X-ray luminosity of $10^{30} \mathrm{erg} \mathrm{s}^{-1} \mathrm{im}$ plies a temperature of $2-3 \times 10^{5} \mathrm{~K}$, while the best fit to the $\mathrm{X}$-ray data gives $10^{6} \mathrm{~K}$. But neither temperature is sufficient to explain the observed optical emission, for which an additional mechanism would be needed. $A$ fortiori the mechanism for the $\gamma$-ray emission, which contains more power than all other wavelengths by a factor of 1,000 , and which was the start of the exercise, remains a mystery.

A. W. Strong is a visiting scientist at the Istituto di Fisica Cosmica e Tecnologie Relative del CNR, Via Bassini 15, 20133 Milan.

\section{Microbiology}

\section{Sublethal attack on bacterial membranes}

from H.R. Perkins

THE membranous layers that envelope cells represent particularly sensitive areas for the destructive action of substances such as antibiotics. For agents to be therapeutically valuable they must, of course, be sufficiently selective to damage the parasite without causing undue harm to the host. An unusual example of such an apparently selective effect, confined to Gram-negative bacteria, is reported in this issue of Naturel.

Bacteria are broadly classified in a binary system according to whether they stain by the procedure described in 1884 by Christian Gram $^{2}$ and are therefore said to be Gram-positive, or are Gram-negative. Work in the ensuing century has shown that these staining characteristics relate to fundamental differences in the organization of the bacterial envelope.

All cells necessarily have a cytoplasmic membrane with a characteristic bilayered structure containing phospholipids and numerous proteins. Outside the membrane, in which the main transport mechanisms of the cell are localized, bacteria generally have a wall layer composed of the specific amino-sugar oligopeptide co-polymer peptidoglycan. Beyond this point the Gram-negative cells differ, in that they have an additional highly specialized outer membrane ${ }^{3,4}$ that limits the passage of hydrophilic molecules of molecular weight greater than about 600 and has specific uptake proteins for many important substances. This outer membrane has the peculiarity that whereas the inner layer of its lipid leaflet contains phospholipids rather like those of the cytoplasmic membrane, the outer lipid layer consists of lipopolysaccharide (L.PS) ${ }^{5}$.

It is the L.PS in the Gram-negative bacterial membrane that seems to be the focus of the initial attack by the membraneactive antibiotic polymyxin B, which consists of a basic cyclic-peptide portion and a short peptide side chain substituted at its $\mathrm{N}$-terminus with a nine-carbon fatty acid. Polymyxin B will interact with bacterial membranes containing L.PS to yield blebs visible under the electron microscope $e^{*}$, and in a recent survey of 35 bacterial species from 27 genera, Wiegel and Quandt ${ }^{7}$ have shown that only bacteria that are biologically 'Gram-negative' will give blebs. (This relationship held even when the results of the Gram stain itself were difficult to interpret, so the procedure should prove very useful in the study of newly isolated bacterial species.)

Nonetheless, the action of polymyxins is not strictly specific and they are toxic to mammalian cells. This toxicity can be reduced by the removal of the fatty acid substituent, but the molecule then also loses its bactericidal activity

In this issue of Nature, Vaara and Vaara ${ }^{1}$ have followed up this research by asking 